\title{
Estequiometría como unidad de aprendizaje en el nivel medio superior del IPN. Análisis desde la docencia
}

Stoichiometry as a learning unit in the Upper Middle Level of the IPN. A teaching analysis

Estequiometria como uma unidade de aprendizagem no nível médio superior do IPN. Análise do ensino Alma Alicia Benítez Pérez
Instituto Politécnico Nacional, Centro de Investigaciones Económicas,
Administrativas y Sociales, CECyT 11, México
abenitez@ipn.mx
https://orcid.org/0000-0001-9213-0379
Aída María Castañeda Rodríguez Cabo
Instituto Politécnico Nacional, Centro de Investigaciones Económicas,
Administrativas y Sociales, México
amcastaneda@ipn.mx
https://orcid.org/0000-0002-5973-7694

Reyes Sánchez Martínez Instituto Politécnico Nacional, Centro de Estudios Científico y Tecnológico Número 8, México rsama45@gmail.com https://orcid.org/0000-0002-0174-2079 


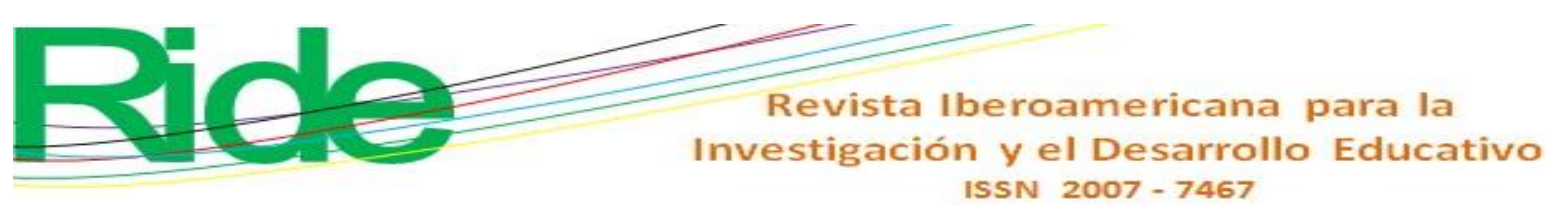

\section{Resumen}

Las estrategias en el proceso de enseñanza-aprendizaje-evaluación (PEAE) tienen un desempeño preponderante. El objetivo central fue analizar a tres docentes que desempeñaron rol de alumnas en el proceso de enseñanza del tema de estequiometría del nivel medio superior en el Instituto Politécnico Nacional (2016). Tradicionalmente, el PEAE se ha centrado en el cálculo y balance de sustancias químicas como estrategia de aprendizaje. Esto genera dificultades en la ejecución de operaciones aritméticas y algebraicas, las cuales son usadas como única forma de aprendizaje, lo que contribuye a los altos índices de desánimo, reprobación y deserción. En este trabajo se utilizó la plataforma Edmodo en cuatro fases: indagación documental, identificación de conocimientos previos, experiencia de una situación no rutinaria, y sentido y significado pedagógico. Los hallazgos muestran autorreflexión de las participantes en su papel como alumnas $y$ en su quehacer docente.

Palabras clave: aprehender, estequiometría, estrategia didáctica, resolución de problemas.

\section{Abstract}

The strategies in the Teaching-Learning-Evaluation Process (PEAE) have a preponderant performance. The main objective was to analyze three teachers in their role as students in this process, in the subject of upper middle level stoichiometry at the National Polytechnic Institute in 2016. Traditionally, the PEAE has focused on the calculation and balance of chemical substances such as learning strategy. This leads to the difficulty in the execution of arithmetic and algebraic operations as the only form of learning and its consequent evaluation, contributes to the high rates of discouragement, disapproval and attrition. The EDMODO platform was used in four phases: documentary inquiry, identification of previous knowledge, experience of a non-routine situation, and pedagogical meaning and meaning. The findings show the teacher's self-reflection in his role as a student and in his teaching work.

Keywords: Apprehend, stoichiometry, didactic strategy, problem solving. 


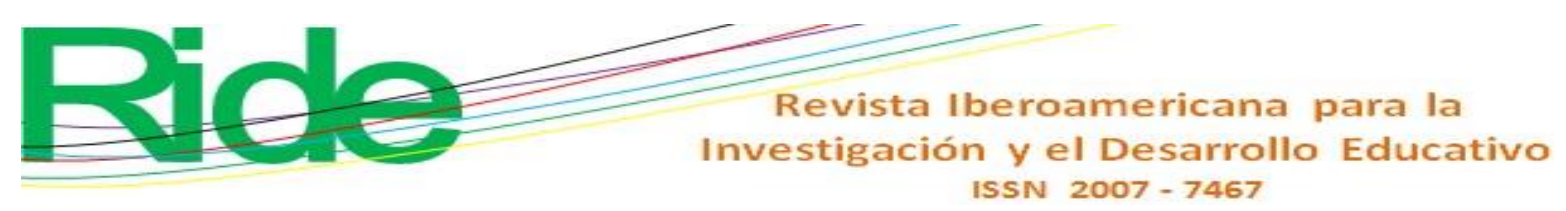

\section{Resumo}

As estratégias no processo de ensino-aprendizagem-avaliação (PEAE) têm desempenho preponderante. $\mathrm{O}$ objetivo principal foi analisar três professores que desempenharam o papel de alunos no processo de ensino da disciplina de estequiometria no ensino médio no Instituto Politécnico Nacional (2016). Tradicionalmente, o PEAE tem se concentrado no cálculo e no equilíbrio de substâncias químicas como uma estratégia de aprendizado. Isso gera dificuldades na execução de operações aritméticas e algébricas, que são usadas como a única forma de aprendizado, o que contribui para os altos índices de desânimo, fracasso e deserção. Neste trabalho, a plataforma Edmodo foi utilizada em quatro fases: pesquisa documental, identificação de conhecimentos prévios, experiência de uma situação não rotineira e significado e significado pedagógico. Os resultados mostram a autorreflexão dos participantes em seu papel como estudantes e em seu ensino.

Palavras-chave: apreender, estequiometria, estratégia didática, resolução de problemas.

Fecha Recepción: Octubre 2019

Fecha Aceptación: Marzo 2020

\section{Introducción}

Todo problema de sustentabilidad ambiental al que nos enfrentamos tiene sus bases en la química y en sus diferentes ramas de conocimiento, entre ellas la estequiometría, palabra cuyo origen se encuentra en los vocablos griegos stoicheion (elemento o parte) y métron (medida para el cálculo de las relaciones cuantitativas entre reactivos y productos en el transcurso de una reacción química). El vasto campo de estudio de esta área del conocimiento está conformado por las reacciones, composición y característica de la materia, aspectos que suelen constituir un reto para los alumnos y, en consecuencia, altos índices de reprobación.

Sobre esta situación, Obando (2013) explica que la enseñanza tradicional de la estequiometría es una de las causas por las que se generan obstáculos en el aprendizaje de los alumnos, mientras que autores como Arasasingham, Taagepera, Potter y Lonjers (2004) señalan que en ciertas prácticas docentes existe una incompleta comprensión de la ecuación química y su relación con la situación empírica. Al respecto, Gabel (1993) destaca que estos obstáculos se deben al énfasis puesto en el nivel simbólico y en la resolución de problemas algorítmicos, en lugar de aquellos fenómenos de la vida cotidiana del alumno. 


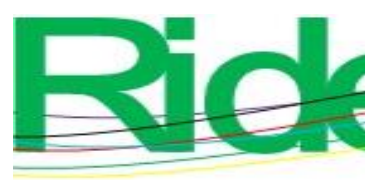

Revista Iberoamericana para la Investigación y el Desarrollo Educativo ISSN 2007 - 7467

Señalado lo anterior, se puede indicar que el objetivo del presente trabajo fue analizar la experiencia de tres docentes del nivel medio superior (NMS) del Instituto Politécnico Superior (IPS) para promover la autorreflexión de su práctica docente en el tema de estequiometría en circunstancias de situaciones no rutinarias relacionadas con el impacto ambiental y la sustentabilidad.

Para lograr dicho objetivo se planteó la siguiente pregunta de investigación: ¿Cómo promover la reflexión del docente en estequiometría a nivel medio superior en el IPN? En esta meta se ha considerado relevante trascender en el proceso de enseñanza-aprendizaje-evaluación (PEAE) para la unidad de aprendizaje estequiometria, al proponer como parte de este proceso temas relacionados con sustentabilidad debido a la relevancia que cobra en el mundo actual. Igualmente, se ha tomado en cuenta que es en ese nivel educativo cuando la enseñanza de las ciencias —en este caso de la estequiometría - exige una concepción pedagógica, didáctica e integral orientada al conocimiento, comprensión y solución de los grandes problemas de sustentabilidad.

\section{Marco de referencia}

En el proceso de enseñanza-aprendizaje-evaluación que todo docente intenta promover subyacen algunos principios fundamentales, uno de los cuales se relaciona con el concepto griego episteme, el cual procura promover el aprendizaje de los alumnos. Esta función es relevante en todas aquellas actividades relacionadas, en primer lugar, con aprender (saber, conocer, descubrir) y, en segundo lugar, con aprehender. Desde el campo de la epistemología, como rama de la filosofía centrada en las posibilidades y limitaciones del conocimiento científico, esta diferencia es notable.

Aprender incluye actividades como estudiar, memorizar, instruir, adquirir competencias para la realización de una actividad, etc. En este marco, el alumno es un sujeto pasivo que transita de un nivel a otro y cuyas acciones no son parte del entorno, pues no lo hace suyo, ya que solo lo incorpora de manera automática. En contraste, el verbo aprehender se vincula con el generar y comprender el conocimiento a partir de una posición personal y/o colectiva frente al medio natural y social. Esta es una postura de adquisición y generación de conocimiento donde el alumno es un ser activo, que aprehende con los sentidos y aplica habilidades y destrezas para comprender la utilidad del aprendizaje de la estequiometría para enfrentar retos de gran relevancia como la escasez del agua, la producción de alimentos y su impacto ambiental, el 


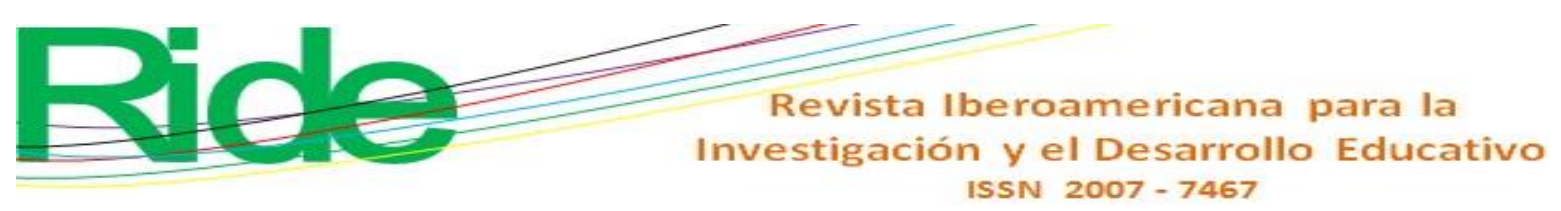

cambio climático, la obtención de energía, etc. El enfoque de ciencia y tecnología desde la sustentabilidad brinda una de las herramientas útiles dentro de los paradigmas pedagógico y didáctico para vincular estos cuatro grandes ejes con cada una de las ciencias químicas.

Aprehender es inherente a toda persona cuando su sentido es claro y adquiere un significado personal que permite responder a las siguientes preguntas: ¿por qué en el mundo actual son necesarios tanto el estudio de la estequiometría como sus múltiples aplicaciones?, ¿para qué realizar cada una de las actividades que implica aprehender este tema?

Además de conocer las múltiples aplicaciones en el contexto científico, tecnológico, social, académico, etc., es necesario, primero, aprender el procedimiento del balance de ecuaciones y, luego, aprehender el tema en el sentido y significado antes mencionados. Esto se logra cuando el discente declara las diferencias entre aprender y aprehender. Este último es un concepto polisémico utilizado en diferentes áreas de conocimiento, tanto para la enseñanza de las ciencias como para las humanidades. A continuación, se verán algunos casos.

Por ejemplo, en el área de la acústica de la física, Boussetta, Beyaoui, Laksimi, Walha y Haddar (2017) afirman: "Furthermore, microscopic observations were realized to better apprehend these damage mechanisms, and to validate the acoustic emission study carried" (p. 175). La polisemia del concepto apprehend conduce a la traducción de captar el entendimiento o capturar su comprensión (entenderlo), mientras que en el Webster Dictionary se lee: “To grasp with the understanding: recognize the meaning of" (p. 55); es decir, lograr su comprensión (comprender el significado de sus elementos).

En el caso de las humanidades, Karim, Mohamed, Ismail y Rahman (2018) utilizan dicho concepto, al cual se le podría traducir como comprensión del fenómeno estudiado. De igual forma, en Younesi y Pirnajmuddin (2018) el concepto apprehends the world se traduce como comprensión del mundo o como calidad de la experiencia de una persona.

En cuanto a la práctica en humanidades, Dallegrave y Burg Ceccim (2018) exponen lo siguiente en un congreso nacional acerca de los programas de residencia en salud:

It is understood that practical learning results from emotional exposure and apprehensions, and that a syllabus is made up of the possibility to apprehend questions and contextualize them, rather than creating a school syllabus based on what will be learned, especially when it comes to in-service education (p. 877).

En este caso, apprehend se traduce como percepción del aprendizaje práctico, resultado de la combinación de elementos perceptivos, emotivos, de sentido y significado que el estudiante 


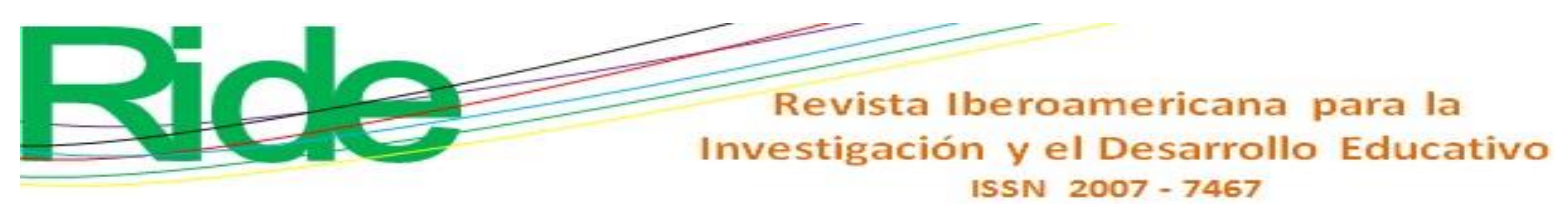

la reflexión crítica de este campo del saber específico. De ahí surge la necesidad de replantearse la generación del conocimiento a través de su mediación, no para aprender (es decir, reiterar, repetir, memorizar), sino para aprehender (inducir, descifrar, contrastar e innovar), y con ello la posibilidad de repensar para construir, y no para consumir de manera pasiva el conocimiento.

\section{Materiales y método}

Etapa 1

El método examinó las siguientes etapas:

Indagación documental. Se realizó la indagación documental respecto al aprendizaje de estequiometría y el proceso enseñanza-aprendizaje de las ciencias. Se comprobó la dificultad en la comprensión de textos científicos, particularmente los relacionados con temas estequiométricos debido a la exigencia de recursos cognitivos necesarios para llevar a cabo tratamientos que permitieran identificar el contenido en el texto (Martínez, 2011). Del mismo modo, se identificó el desinterés de los alumnos por aprender ciencia, situación motivada por la enseñanza tradicional recibida en los niveles educativos anteriores (Obando, 2013). A ello se suma el escaso conocimiento previo necesario para iniciar el aprendizaje de estequiometría, con competencias matemáticas débiles o nulas tales como relaciones porcentuales, razones y proporciones, factores de conversión y de química, nomenclatura de elementos y compuestos, balanceo de reacciones químicas (Valderrama y Gonzales, 2010). Finalmente, la literatura reporta las dificultades que enfrentan los alumnos de nivel bachillerato en su curso de química respecto a un lenguaje químico altamente simbólico y formalizado, lo que conlleva un aprendizaje no significativo (Obando, 2013).

Respecto a la enseñanza de las ciencias, Marcelo (2009) considera el desarrollo profesional docente como un proceso colaborativo, relacionándolo con las reformas escolares como escenarios idóneos para reflexionar el quehacer de aula. En este sentido, Ponte (1998) menciona diversos aspectos para llevar cabo el desarrollo profesional: discusión permanente con sus homólogos, toma de decisiones, intercambio de vivencias en el aula y actualización disciplinar. Para Perrenoud (2000) la formación de docentes debe impulsar el desarrollo de competencias que involucren saberes disciplinarios y metodológicos, además de sistematizar situaciones de aprendizaje, facilitar y organizar su progreso, implicar de manera activa a los alumnos, implementar el uso de tecnologías y fomentar el trabajo colaborativo. 


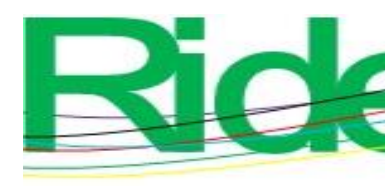

Revista Iberoamericana para la
Investigación y el Desarrollo Educativo
ISSN $2007-7467$

En esta dirección, Day (2001) señala diversas metas en la formación del docente y exhorta la continuidad de saberes de la profesión desde una visión planeada para lograr fines determinados. En el contexto de aula, Day menciona metas dirigidas al desarrollo científico y pedagógico, reflexión permanente sobre su quehacer educativo y actualización constante sobre las unidades de aprendizaje.

De esta forma, el desarrollo profesional del docente se reconoce como proceso de producción de saberes para impulsar el diálogo con sus homólogos en temas referidos a la actividad docente. En este contexto, la actualización en conocimientos científicos se hace necesaria, así como la articulación con otras áreas del conocimiento en temas actuales de la ciencia con impacto social (por ejemplo, problematización y contextualización, resolución de problemas, enseñanza desde la investigación, uso de tecnologías de información y comunicación, etc.).

El docente debe recontextualizar la pedagogía al contenido específico a favor del aprendizaje del estudiante (Acevedo-Díaz, 2009) articulándolo con otras áreas del saber. Ello implica la elaboración de estrategias, material didáctico y prácticas innovadores para motivar al estudiante e involucrarlo en las problemáticas actuales de impacto social.

\section{Etapa 2}

Conocimientos previos. Se diseñó un instrumento diagnóstico con la finalidad de identificar los conocimientos previos necesarios para el aprendizaje de estequiometría, el cual se aplicó a las docentes participantes. Para ello, se consideró llevar a cabo los pasos recomendados por Luchetti y Berlanda (1998), quienes evidencian las capacidades cognitivas de los participantes. El instrumento fue diseñado con base en el contenido curricular de la materia Química II. Se identificaron cuáles fueron los conocimientos necesarios y suficientes para equiparar los saberes previos. En la tabla 1 se establecen los contenidos programáticos en estequiometría y los conocimientos previos antes de iniciar el tema. 


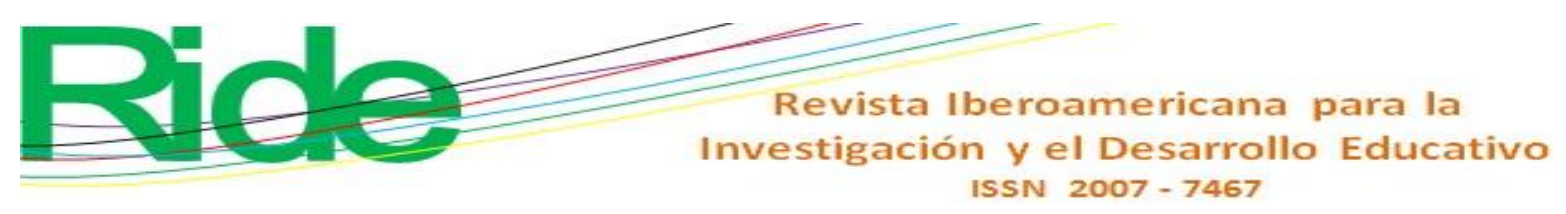

Tabla 1. Contenido programático de estequiometría y conocimientos previos necesarios

\begin{tabular}{|c|c|c|}
\hline N. ${ }^{\circ}$ & Contenido programático & Conocimientos previos necesarios \\
\hline 1 & $\begin{array}{l}\text { Determinación de la fórmula } \\
\text { mínima de un compuesto }\end{array}$ & $\begin{array}{l}\text { Cálculo aritmético de porcentajes de cantidades } \\
\text { Conocimiento de tabla periódica de los elementos } \\
\text { Concepto de peso atómico } \\
\text { Concepto de peso molecular }\end{array}$ \\
\hline 2 & $\begin{array}{l}\text { Leyes ponderales: } \\
\text { Ley de conservación de la } \\
\text { materia. } \\
\text { Cálculos estequiométricos de } \\
\text { reacciones químicas }\end{array}$ & $\begin{array}{l}\text { Conocimiento de tabla periódica de los elementos } \\
\text { Concepto de peso atómico } \\
\text { Concepto de peso molecular } \\
\text { Reacción química } \\
\text { Balanceo de reacciones químicas } \\
\text { Regla de tres simple o factores de proporción }\end{array}$ \\
\hline 3 & Eficiencia de una reacción & $\begin{array}{l}\text { Cálculo aritmético de porcentajes de cantidades } \\
\text { Concepto de peso molecular } \\
\text { Conocimiento de tabla periódica de los elementos } \\
\text { Concepto de peso atómico } \\
\text { Concepto de peso molecular } \\
\text { Reacción química } \\
\text { Balanceo de reacciones químicas } \\
\text { Regla de tres simple o factores de proporción }\end{array}$ \\
\hline 4 & Pureza de los reactivos & $\begin{array}{l}\text { Cálculo aritmético de porcentajes de cantidades } \\
\text { Concepto de peso molecular }\end{array}$ \\
\hline 5 & $\begin{array}{l}\text { Reactivo límite y reactivo en } \\
\text { exceso }\end{array}$ & $\begin{array}{l}\text { Conocimiento de tabla periódica de los elementos } \\
\text { Concepto de peso atómico } \\
\text { Concepto de peso molecular } \\
\text { Reacción química } \\
\text { Balanceo de reacciones químicas } \\
\text { Regla de tres simple o factores de proporción }\end{array}$ \\
\hline
\end{tabular}

Fuente: Elaboración propia con base en Karim et al. (2018)

El instrumento contó con 25 problemas de opción múltiple: 10 reactivos de razones y proporciones y regla de tres simple, 5 de cálculo de porcentaje, 5 de nomenclatura de compuestos químicos y 5 de balance de reacciones químicas. A continuación, se muestran dos ejemplos de 


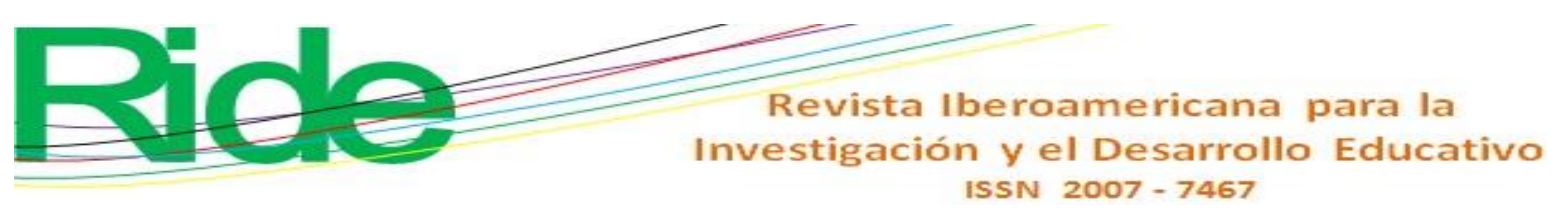

reactivos referentes a proporciones directa e inversa, regla de tres simple y balance de reacciones químicas.

- La descomposición de $36 \mathrm{~g}$ de agua produce $4 \mathrm{~g}$ de hidrógeno gaseoso. ¿Cuánto hidrógeno gaseoso se produce cuando se descomponen $180 \mathrm{~g}$ de agua?
a. $200 \mathrm{~g}$
b. $20 \mathrm{~g}$
c. $0.80 \mathrm{~g}$
d. $0.20 \mathrm{~g}$

Balancea la reacción siguiente seleccionando los coeficientes correspondientes en cada compuesto:
e. $2,13,8,10$
f. $2,8,13,10$
g. $1,6,4,5$
h. $10,8,13,2$

$$
\mathrm{C}_{4} \mathrm{H}_{10}+\mathrm{O}_{2} \rightarrow \mathrm{CO}_{2}+\mathrm{H}_{2}
$$

La aplicación del instrumento se realizó en la plataforma educativa Edmodo.

\section{Etapa 3}

Experiencia de una situación no rutinaria. Se impulsó la resolución de problemas con el propósito de que los tres docentes vivieran la experiencia como alumnos y reflexionaran acerca de su desempeño como profesores en la enseñanza de la estequiometría frente a situaciones no rutinarias.

El aprendizaje contextualizado y/o aprehendizaje contextualizado desde la resolución de problemas en ciencias favorece el aprendizaje de concepto (Cala, Mariño y Casas, 2009), así como el desarrollo de habilidades y destrezas para enfrentar situaciones no rutinarias, lo que demanda reflexión, búsqueda de información, constante cuestionamiento y toma de decisiones para emitir una respuesta analizada y definida, una estrategia de solución.

Para el presente estudio se determinó la clasificación de problemas desarrollada por Wakefield (1992), particularmente los problemas semiabiertos y semicerrados. Los primeros son aquellos parcialmente definidos donde se identifican algunos parámetros y la solución presenta diversas posturas, es decir, más de una respuesta. Los segundos están parcialmente definidos y presentan una única repuesta (Wakefield, 1992). 


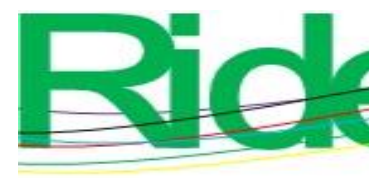

\section{Revista Iberoamericana para la Investigación y el Desarrollo Educativo ISSN $2007-7467$}

El problema de la experiencia ofrecida pertenece a problemas semicerrados debido al número de participantes, lo cual permitió búsqueda de información y discusión continua. Durante la experiencia, se promovió el desarrollo de procedimientos científicos (observar, inferir, explicar, argumentar, etc.) en ambientes consensuados de reflexión y análisis para así impulsar la toma de decisiones durante el proceso de solución.

El problema seleccionado fue adaptado de un ejercicio del artículo El trabajo práctico integrado con la resolución de problemas y el aprendizaje conceptual en la química de los polímeros (Garritz e Irazoque, 2004). La situación contempla el análisis de los conceptos reactivo límite y reactivo en exceso en una reacción química, dentro del dominio de la estequiometría. Además, atiende una problemática socioambiental: los polímeros. Se consideró su aplicación para la fabricación de artículos de consumo y el proceso de obtención del nailon 6-10, así como para el análisis de los conceptos de inmiscibilidad, densidad, tipos de reacción, grupos funcionales de química orgánica. Con ello, se procuró que el participante interviniera desde una postura crítica para emitir soluciones analizadas y argumentadas en contextos consensuales. El texto de la situación es el siguiente:

- En el ámbito industrial la fabricación de nailon 6-10 se realiza a partir de la reacción de una diamina y un ácido carboxílico, aunque el proceso es muy lento. En el laboratorio podría acelerarse la reacción utilizando la hexametilen diamina y el cloruro de sebacoilo (Garritz y Irazoque, 2004, p. 47).

La situación contextualizada contó con nueve preguntas guía para llevar a cabo la actividad (tabla 2); el cuestionario expone la intención de estas preguntas para identificar posibles conexiones con los conocimientos previos y los nuevos conceptos, el empleo de analogías y el uso de estrategias para su solución, así como el impacto de los polímeros en el medio ambiente. 


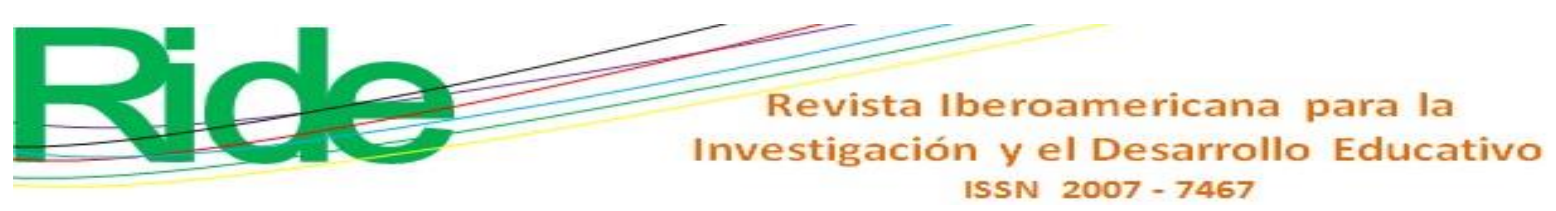

Tabla 2. Preguntas guía del problema propuesto

\begin{tabular}{|c|c|}
\hline & \\
\hline $\begin{array}{l}\text { 1. Explica brevemente si técnicamente es } \\
\text { posible realizar el experimento para la } \\
\text { obtención del hilo nailon 6-10 en el } \\
\text { laboratorio, de modo que los alumnos } \\
\text { observen la polimerización claramente. } \\
\text { Describe el procedimiento para realizarla, } \\
\text { considerando la hexametilendiamina y el } \\
\text { cloruro de sebacoilo }\end{array}$ & $\begin{array}{l}\text { Explicación para realizar el experimento de } \\
\text { obtención del nailon 6-10. } \\
\text { Se identifican habilidades para investigar, } \\
\text { así como curiosidad, creatividad, habilidad } \\
\text { para indagar, seleccionar y clasificar } \\
\text { información. }\end{array}$ \\
\hline $\begin{array}{l}\text { 2. Si se requiere obtener } 150 \mathrm{~g} \text { de nailon } 6-10 \text {, } \\
\text { ¿cuáles serán las cantidades de los reactivos } \\
\text { si la reacción tiene una eficiencia de } 90 \% \text { ? }\end{array}$ & $\begin{array}{l}\text { Se solicita al docente las cantidades de } \\
\text { reactivos necesarias para obtener } 150 \mathrm{~g} \text { de } \\
\text { nailon 6-10, considerando una eficiencia de } \\
\text { reacción de } 90 \% \text {. Aplicación de los } \\
\text { conceptos estequiométricos: ley de } \\
\text { conservación de la materia, balanceo de } \\
\text { ecuaciones químicas y concepto de } \\
\text { rendimiento de la reacción. } \\
\text { Se requiere investigación previa de la } \\
\text { reacción química por realizar. } \\
\text { Conocimientos previos: proporciones con } \\
\text { regla de tres, factores de relación molar, } \\
\text { rendimiento teórico, rendimiento real y } \\
\text { rendimiento porcentual. Empleo de } \\
\text { analogías. }\end{array}$ \\
\hline $\begin{array}{l}\text { 4. ¿Qué sucede cuando una de las aminas de la } \\
\text { hexametilendiamina reacciona con un cloro } \\
\text { del cloruro de sebacoilo para formar cloruro } \\
\text { de hidrógeno y el segundo cloro de la misma } \\
\text { molécula reacciona con otra molécula de } \\
\text { amina y así sucesivamente? }\end{array}$ & $\begin{array}{l}\text { Explicar claramente el experimento } \\
\text { práctico en el laboratorio y describir lo } \\
\text { observado (la polimerización forma una } \\
\text { cadena que es el hilo de nailon). }\end{array}$ \\
\hline
\end{tabular}




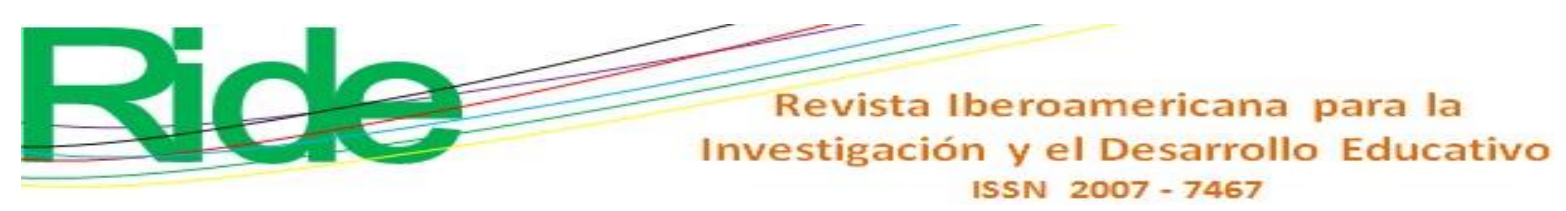

\section{Resultados}

Los resultados arrojaron buen nivel de dominio por los docentes en la solución de problemas de estequiometría; no obstante, en la sección de razones y proporciones presentaron errores en su planteamiento, principalmente porcentajes de nomenclatura y balance de reacciones. Se decidió denominar categoría I al uso de procesos para la significación de contenidos por los docentes (tabla 3), la cual está orientada hacia la identificación de elementos relevantes (o significativos) en la situación problemática para analizar tratamientos en la información y reconocer su articulación. Esto otorga la posibilidad de establecer nuevas oportunidades en la solución del problema, en la aplicación de procesos químicos para la significación de contenidos por los docentes.

Tabla 3. Indicadores categoría I

\begin{tabular}{|l|l|}
\hline ER & Se Explicitan los elementos Relevantes o significativos en la situación. \\
\hline TIE & $\begin{array}{l}\text { Tratamiento de la Información; cálculos Estequiométricos orientados a la acción } \\
\text { sobre la base de las relaciones establecidas. }\end{array}$ \\
\hline AI & Articulación de la Información en el contexto situacional. \\
\hline
\end{tabular}

Fuente: Elaboración propia con base en Garritz e Irazoque (2004)

El diseño de una estrategia de enseñanza debe iniciar con la vivencia de diversas experiencias y permitir al docente advertir retos, obstáculos y fortalezas que el alumno enfrentará día tras día en el aula. Debido a ello, la categoría II se enfoca en la autorreflexión del docente ante su experiencia para repensar estrategias didácticas en el quehacer diario en el aula (tabla 4).

Tabla 4. Indicadores categoría II

\begin{tabular}{|l|l|}
\hline QQ & ¿Qué y para Qué? \\
\hline HT & Herramientas TIC. Edmodo. \\
\hline EE & Estrategias para aprehender temas Estequiométricos. \\
\hline
\end{tabular}

Fuente: Elaboración propia con base en Karim et al. (2018)

Se emplea un método de análisis proveniente del campo de las ciencias sociales. Se evidencia el desempeño del docente durante la experiencia, estableciendo los mecanismos utilizados para solucionar la situación problemática. A continuación, se expone el análisis correspondiente a la categoría I (tabla 5) y posteriormente el análisis de la categoría II (tabla 6). 


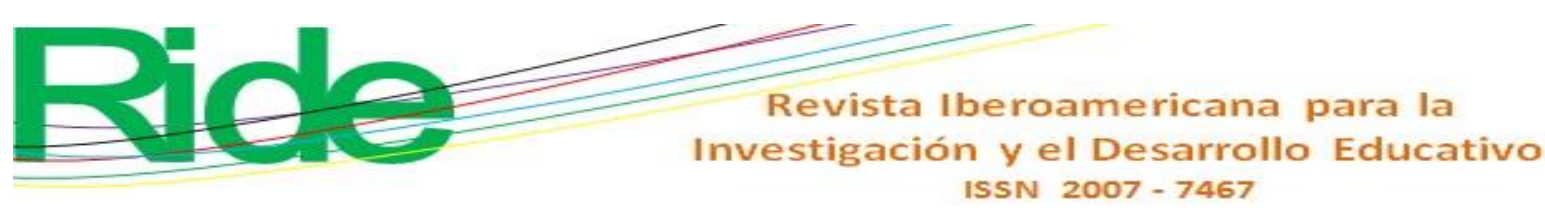

Tabla 5. Categoría I. Aplicación de procesos químicos para la significación de contenidos por los docentes.

\begin{tabular}{|c|c|}
\hline \multirow[t]{3}{*}{ ER } & $\begin{array}{l}\text { Docente A: Indica paso a paso la técnica de laboratorio para la obtención de nailon } \\
\text { 6-10, aunque presenta errores en el balanceo. }\end{array}$ \\
\hline & B: Explica el procedimiento para obtener el nailon 6-10. \\
\hline & $\begin{array}{l}\text { Docente C: Presenta de manera general necesidades de materiales, reactivos; } \\
\text { control de las condiciones de reacción, sin especificaciones. }\end{array}$ \\
\hline \multirow[t]{3}{*}{ TIE } & $\begin{array}{l}\text { Docente A: El cálculo es correcto para la cantidad de reactivo de } \\
\text { hexametilendiamina, aplicando las relaciones estequiométricas. }\end{array}$ \\
\hline & $\begin{array}{l}\text { Docente B: Establece las relaciones ponderales que se deben realizar para el } \\
\text { cálculo estequiométrico de cada reactivo y el ajuste debido al rendimiento de } 90 \% \\
\text { de la reacción. }\end{array}$ \\
\hline & $\begin{array}{l}\text { Docente C: Escribe la reacción sin balancear, con errores en los nombres de los } \\
\text { reactivos debajo de su fórmula en la reacción. }\end{array}$ \\
\hline \multirow[t]{3}{*}{ AI } & $\begin{array}{l}\text { Docente A: No establece con precisión que el reactivo limitante es el cloruro de } \\
\text { sebacoilo, además trata de establecer una cadena polimérica. }\end{array}$ \\
\hline & $\begin{array}{l}\text { Docente B: Explica la combinación de los dos reactivos: los extremos del } \\
\text { hexametilendiamina reaccionan con el cloruro de sebacoilo para formar nuevos } \\
\text { enlaces de amida, creándose una cadena alternada de los dos grupos funcionales } \\
\text { diferentes para producir un polímero denominado nailon 6-10. }\end{array}$ \\
\hline & $\begin{array}{l}\text { Docente C: No expone el procedimiento, solo menciona que ocurre la } \\
\text { polimerización por condensación. }\end{array}$ \\
\hline
\end{tabular}

Fuente: Elaboración propia con base en Garritz e Irazoque (2004) 


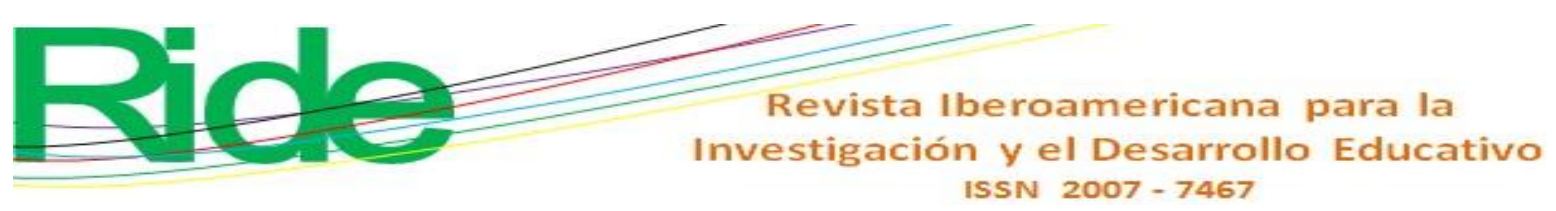

Tabla 6. Categoría II. Repensar estrategias didácticas

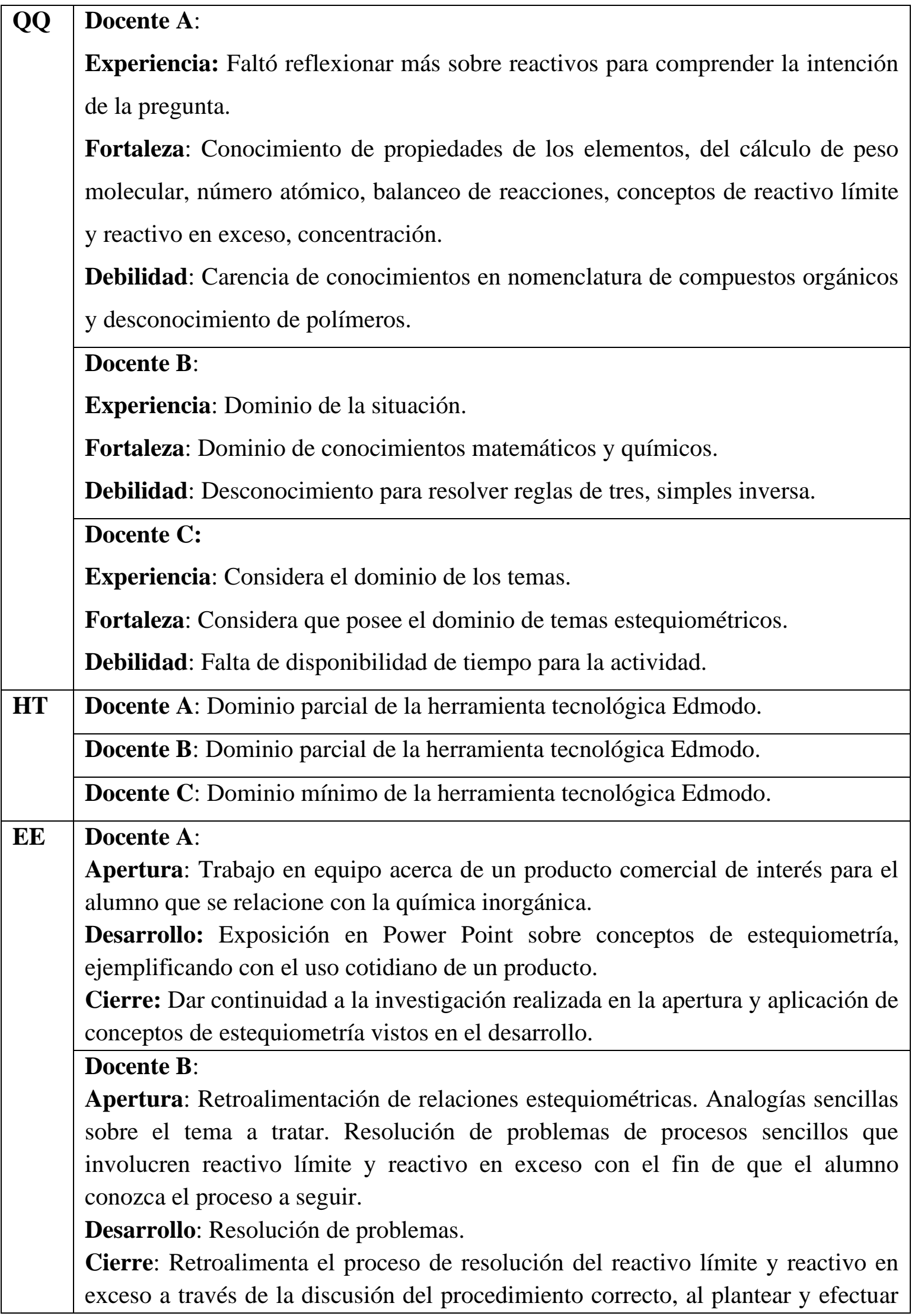




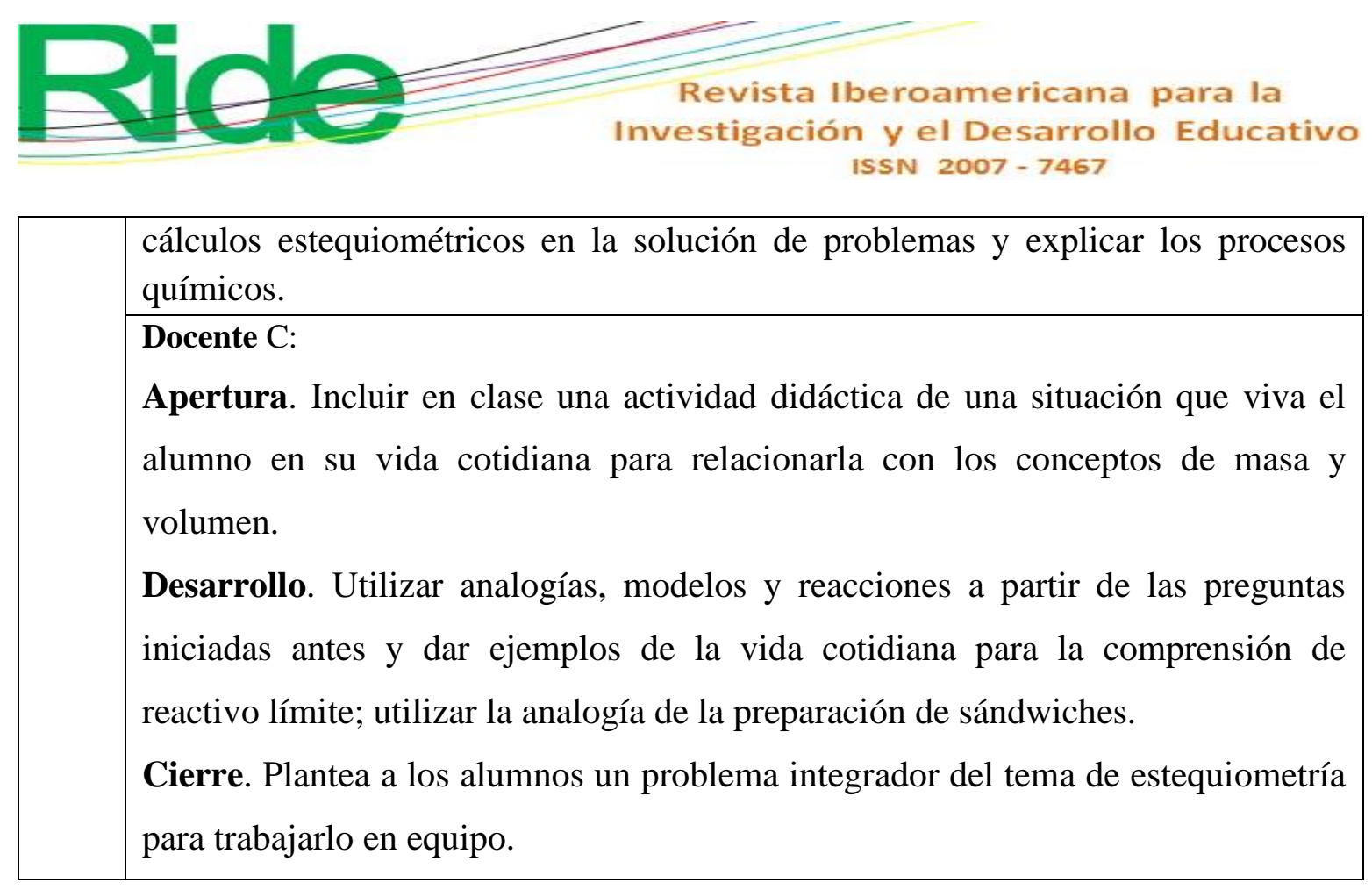

Fuente: Elaboración propia con base en Martínez 2011

Finalmente, como resultado de investigación, en nosotros se dio un cambio: ahora tenemos la firme convicción de la necesidad de "atrevernos a saber" como herramienta vital de nuestro quehacer docente para que se dé la ilustración, es decir, "la libertad de hacer" o —en palabras de Emanuel Kant_- “el uso público de la razón”.

\section{Discusión}

La intención de esta investigación fue evidenciar la importancia de la enseñanza de las ciencias (en este caso, de estequiometria) en el NMS, proceso que de acuerdo con la disertación expuesta requiere una concepción pedagógica, didáctica e integral orientada al conocimiento, comprensión y solución de problemas reales (Valderrama y Gonzales, 2010). A continuación, se expone la discusión de los indicadores con base en el análisis organizado y sistemático desarrollado. 


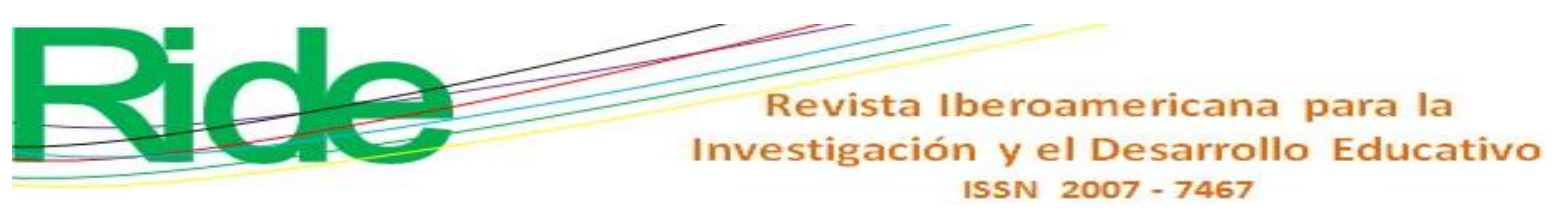

\section{Categoría I. Aplicación de procesos químicos para la significación del contenido por los docentes Indicador ER}

Las docentes A y B identificaron información relevante en el contexto de la situación y utilizaron fuentes de información existentes en la Web para describir la forma práctica de obtener en el laboratorio el nailon 6-10 y la reacción química que ocurre. Referente a la estequiometría, balanceo de reacción, rendimiento de la reacción y reactivo límite, se resolvieron satisfactoriamente a través de la articulación de la información obtenida.

El docente $\mathrm{C}$ no logró identificar información relevante para la obtención del nailon y presentó errores en la reacción química, así como desaciertos en la conceptualización y cálculos estequiométricos para el reactivo límite y rendimiento.

\section{Indicador TIE}

Los docentes no lograron establecer la relación adecuada durante el experimento y, con ello, explicar los conceptos de densidad al observar que un líquido se aloja en la parte superior del recipiente y otro en la inferior debido a sus diferentes densidades; la inmiscibilidad que se observa aun cuando se agitan para mezclarlos y que se separan después de un tiempo.

Asimismo, las fórmulas permiten identificar grupos funcionales de los compuestos orgánicos, lo cual no fue visualizado por los docentes al no representar sentido alguno en su quehacer, como se aprecia en las respuestas de los docentes A y C, respectivamente.

Docente A: No tenía conocimiento de nomenclatura de compuestos orgánicos y los polímeros tienen muchas moléculas. Como alumno desconocía el nombre de los compuestos mencionados, pero los investigué en Internet y encontré la fórmula de cada uno de ellos.

Docente C: En un principio con algo de incertidumbre. Un poco desorientado por no conocer las fórmulas de los reactivos ni las cantidades de los mismos, necesarios para resolver la pregunta del reactivo límite. Además, por tratarse de una reacción de polimerización que no es común para estequiometría.

\section{Indicador AI}

El docente A articuló la información para la polimerización por condensación, pero no argumentó su respuesta. Por otro lado, el docente B articuló la información y emitió argumentos para la cadena polimérica, además consideró que es posible observar cómo se forma una gran 


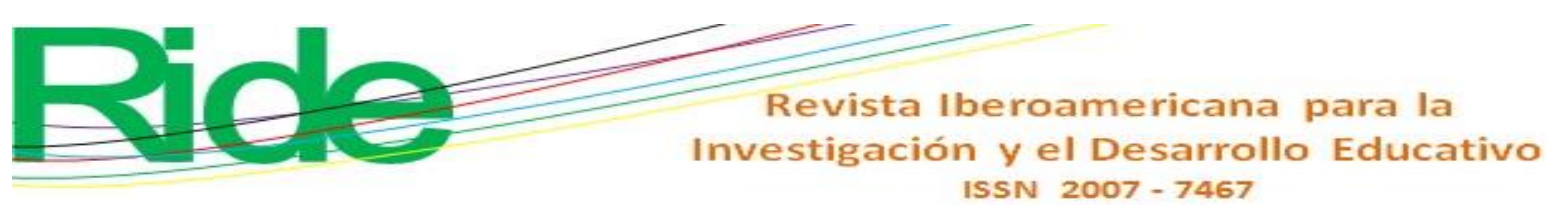

molécula cuando se va separando el hilo de nailon de la interface de los dos líquidos en el experimento. El docente $\mathrm{C}$ no estableció ninguna articulación durante su disertación, dado que no le representó ningún significado.

\section{Categoría II. Repensar estrategias didácticas \\ Indicador QQ}

Los docentes consideraron que la resolución de problemas es adecuada para ser aplicada con los alumnos, puesto que ellos se sintieron bien al resolver la actividad. No obstante, el docente A comentó que en algunas preguntas faltó reflexionar más sobre el contenido.

El docente A expresó la ausencia de obstáculos al resolver el problema, en tanto que el docente $\mathrm{C}$ sí enfrentó dos obstáculos: la falta de disponibilidad de tiempo y de conocimiento de la plataforma educativa, mientras que el docente B expresó la necesidad de recordar la forma de resolver reglas de tres de proporción inversa.

En cuanto a las fortalezas, los tres docentes indicaron que se enfocan en el dominio de contenidos en su materia y de conceptos matemáticos; sin embargo, el docente $\mathrm{C}$ comentó la presencia de errores con sus alumnos en la solución de razones y proporciones en matemáticas y química.

Para resolver la actividad del problema de estequiometría, la docente A expuso cierta incertidumbre en cuanto a la ecuación que representa la reacción de polimerización y cálculo del número de moléculas en el polímero. Por su parte, la docente B indicó que su obstáculo fue el desconocimiento de las fórmulas de los compuestos.

\section{Indicador HT}

En cuanto al manejo de la plataforma educativa Edmodo, los docentes A y B comentaron que no tuvieron problema alguno, en tanto que el docente $\mathrm{C}$ sí tuvo problemas para manejarla, por lo que experimentó incomodidad e incertidumbre, aunque reconoció la gran utilidad del recurso para apoyar las actividades académicas.

Referente a la problemática para la obtención del nailon, los tres docentes confesaron su desconocimiento de las fórmulas de los reactivos: cloruro de sebacoilo y hexametilendiamina. Los docentes A y B recurrieron de inmediato a los recursos web para conocer sus fórmulas, así como la reacción que se lleva a cabo para la obtención del nailon 6-10. La información proporcionada en la biblioteca de la plataforma Edmodo facilitó la indagación en la situación. El 


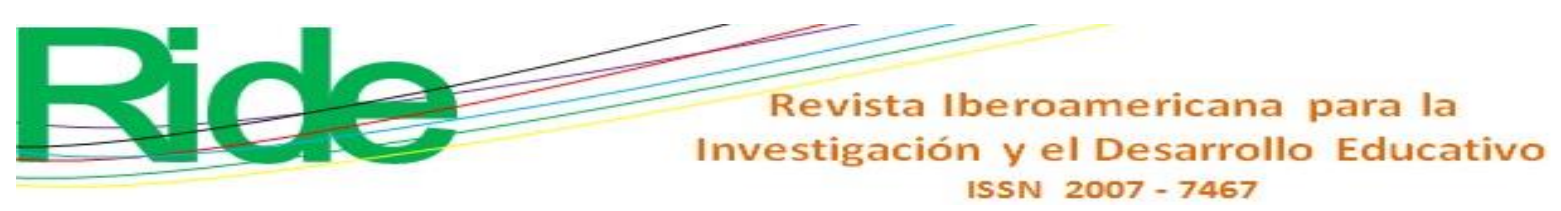

docente $\mathrm{C}$ enfrentó más dificultades para conseguir la información porque desconocía cómo emplear la plataforma.

\section{Indicador EE}

Desde una visión pedagógica, el docente B manifestó la importancia de impulsar el aprendizaje significativo de los alumnos en el tema de estequiometría empleando la misma secuencia de actividades de esta investigación, es decir, adecuar al inicio del curso un proyecto elegido por los alumnos para indagar y seleccionar la información, elaborar mapas conceptuales y exposición del trabajo. El docente consideró una secuencia didáctica de apertura, desarrollo y cierre, aunque ignora el recurso de saberes previos. El docente A expuso el planteamiento de problemas en contextos de la vida real e impulsó la indagación por parte de los alumnos sobre conceptos de estequiometría. Finalmente, creó espacios de reflexión entre los equipos para discutir las propuestas en un ambiente consensuado. Cabe mencionar la ausencia de las TIC en su propuesta.

El docente $\mathrm{C}$ incluyó actividades de apertura generales y dirigió su intervención durante el desarrollo para concluir con una actividad integradora. Se percibió una enseñanza centrada en el docente con el alumno como receptor. La propuesta del docente C no incluyó la identificación de saberes previos del alumno; combinó los tres tiempos: apertura, desarrollo y cierre, y se evidenció la falta de actividades didácticas y ausencia del uso de recursos web.

\section{Conclusiones}

Los docentes con estudios superiores en química deben fortalecer su formación como facilitadores en unidades de aprendizaje como la estequiometría, pues sus estrategias didácticas son escasas, hecho que fue evidente al modificar dicho tema. Su quehacer como docentes está centrado en estrategias de aprendizaje que provocan que el alumno sea un discente pasivo. De hecho, los docentes participantes reproducen los métodos de aprendizaje que adquirieron durante su formación profesional, especialmente con actividades hasta cierto punto mecánicas. Esto quedó demostrado al aplicar a los docentes el ejercicio de un tema no rutinario, como es el caso de producción de polímeros, cuyo impacto en el medio ambiente es nocivo. 


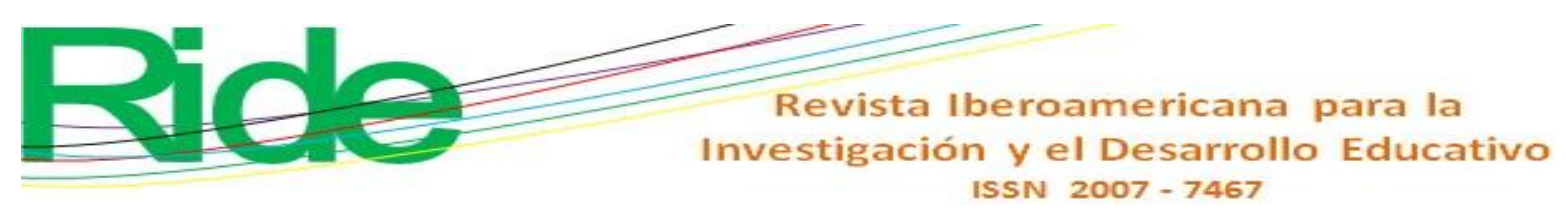

Los datos obtenidos, por tanto, muestran la necesidad de discutir y proponer en las academias, y a nivel curricular, una concepción epistemológica distinta del verbo aprender para actualizarlo por el verbo aprehender, un concepto más polisémico, como quedó expresado líneas arriba.

El docente es un factor fundamental para avanzar hacia la mejora de la enseñanza de la química en el nivel medio superior, por lo que es necesaria su formación didáctica y pedagógica; además de su compromiso y actualización de su práctica docente con estrategias novedosas que incluyan herramientas tecnológicas.

En definitiva, se puede concluir que existe un amplio campo de oportunidad para los docentes de química con la aplicación de las TIC y con en el diseño de actividades didácticas que resulten atractivas para los alumnos, lo que podrá promover un aprendizaje más significativo.

\section{Agradecimientos}

Los autores agradecen el apoyo brindado por el Instituto Politécnico Nacional, a través de la Secretaría de Investigación y Posgrado, para realizar esta investigación registrada con en el número: 20171093. Asimismo, a todos los académicos que intervinieron. 


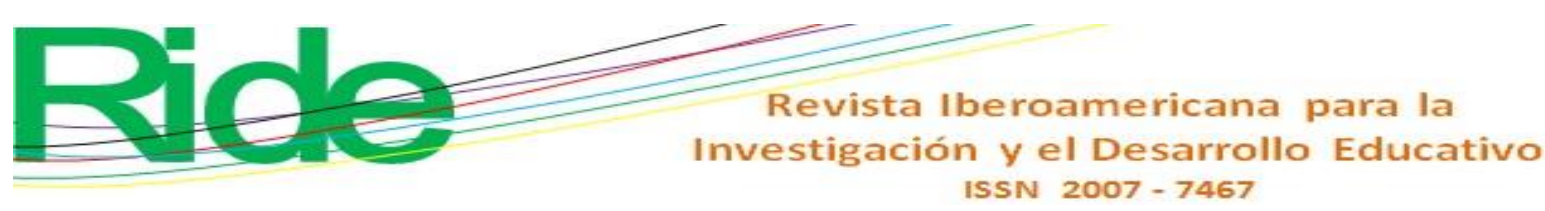

Gil, D. (1998). El papel de la educación ante las transformaciones científicas y tecnológicas. Revista Iberoamericana de Educación, 18(1), 69-90.

Hrich, N., Lazaar, M. and Khaldi, M. (2019). Improving Cognitive Decision-Making into Adaptive Educational System through a Diagnosis Tool based on the Competency Approach. International Journal of Emerging Technologies in Learning, 14(7), 226-235. doi:https://online-journals.org/index.php/i-jet/article/view/9870

Karim, A., Mohamed, A. R., Ismail, S. and Rahman, M. M. (2018). Organized Hypocrisy in EFL Teacher Training Programs. International Journal of Instruction, 11(2), 437-450. Doi: https://doi.org/10.12973/iji.2018. 11230a

Luchetti, E. L. y Berlanda, O. G. (1998). El diagnóstico en el aula. Buenos Aires, Argentina: Magisterio del Río de la Plata.

Maldaner, O. A. (2000). A Formação Inicial e Continuada de Professores de Química: professores/pesquisadores. Ijuí, RS: Ed. UNIJUI.

Marcelo, C. (2009). Desenvolvimento Profissional Docente: passado e futuro. Sísifo. Revista de Ciências da Educação, (8), 7-22.

Martínez, L. F. (2009). Enseñanza constructivista sobre conceptos de cinética en la formación inicial de profesores de química. Educación Química, 20(3), 283-392. Doi: http://dx.doi.org/10.1016/S0187-893X(18)30040-5.

Martínez, M. (2011). Identificación y categorización de las dificultades en la lectura y comprensión de los enunciados de problemas de estequiometría en Química Aplicada (tesis de maestría). Universidad Nacional de Córdoba, Argentina.

Mellado, V. (1996). Concepciones y prácticas de aula de profesores de ciencias, en formación inicial primaria y secundaria. Enseñanza de las Ciencias, 14(3), 289-302.

Mellado, V. y González, T. (2000). La formación inicial del profesorado de ciencias. En Perales, F. y Cañal, P. (comps.), Didáctica de las ciencias experimentales: teoría y práctica de la enseñanza de las ciencias (pp. 535-555). España: Marfil.

Mellado, V. y González, T. (2000). La formación inicial del profesorado de ciencias. En Perales, F. J. y Cañal, P. (eds.), Didáctica de las Ciencias Experimentales. Alcoy: Marfi.

Obando, S. (2013). Implementación de estrategias didácticas para la enseñanza de la estequiometría en estudiantes del grado once de enseñanza media (tesis maestría). Universidad Nacional de Colombia, Colombia. Recuperado de http://bdigital.unal.edu.co/10308/1/36758490.2013.pdf. 


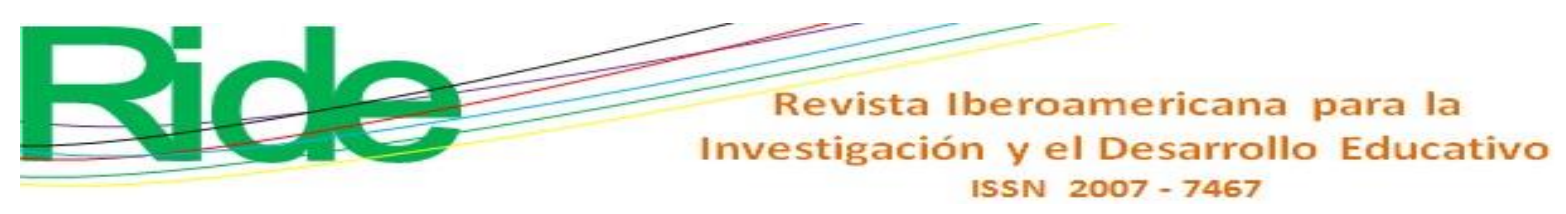

Perrenoud, P. (2000). Dez novas competências para ensinar. Porto Alegre: Artes Médicas Sul. Recuperado de https://www.unige.ch/fapse/SSE/teachers/perrenoud/php_main/OUVRAGES/Perrenoud_ 2000_A.html.

Ponte, J. P. (1998). Da formação ao desenvolvimento profissional. En Actas do ProfMat 98. Lisboa: Associação dos Professores de Matemáticas, 27-44.

Schulman, I. (1987). Knowledge and teaching: foundations of the new reforms. Harvard Educational Review, 57(1), 1- 22.

Valderrama, A. C. y Gonzales, P. E. (2010). En busca de alternativas para facilitar la enseñanzaaprendizaje de la estequiometría. En Blanco \& Negro, 1(1). Recuperado de http://revistas.pucp.edu.pe/index.php/enblancoynegro/article/view/2186/2117.

Wakefield, J. (1992). Creative thinking: Problem solving skills and the arts orientation. Norwood, N. J.: Ablex Publishing. 140. Doi: https://doi.org/10.1080/10400418909534300 Younesi, M. and Pirnajmuddin, H. (2018). Nostalgia and the Sublime in Comarc McCarthy's. The Border Trilogy. Atlantis. Journal of Spanish Association of Anglo-American Studies, 45-62. Doi: http://doi.org/10.289/Atlantis-2018-40.2.03 


\begin{tabular}{|c|c|}
\hline Rol de Contribución & Autor (es) \\
\hline Conceptualización & $\begin{array}{l}\text { Alma Alicia Benítez Pérez (principal) y Reyes Sánchez } \\
\text { Martínez (igual) }\end{array}$ \\
\hline Metodología & Alma Alicia Benítez Pérez \\
\hline Software & $\begin{array}{l}\text { Programación, desarrollo de software; Diseño de programas } \\
\text { informáticos; Implementación del código informático y } \\
\text { algoritmos de soporte; Pruebas de componentes de código } \\
\text { existentes. }\end{array}$ \\
\hline Validación & $\begin{array}{l}\text { Alma Alicia Benítez Pérez (principal), Reyes Sánchez } \\
\text { Martínez (igual) y Aída María Castañeda Rodríguez Cabo } \\
\text { (apoyo) }\end{array}$ \\
\hline Análisis Formal & $\begin{array}{l}\text { Alma Alicia Benítez Pérez(principal), Reyes Sánchez Martínez } \\
\text { (igual) y Aída María Castañeda Rodríguez Cabo (apoyo) }\end{array}$ \\
\hline Investigación & $\begin{array}{l}\text { Reyes Sánchez Martínez (principal), Alma Alicia Benítez } \\
\text { Pérez (igual) y Aída María Castañeda Rodríguez Cabo (apoyo) }\end{array}$ \\
\hline Recursos & $\begin{array}{l}\text { Reyes Sánchez Martínez (principal) y Alma Alicia Benítez } \\
\text { Pérez (igual) }\end{array}$ \\
\hline Curación de datos & $\begin{array}{l}\text { Reyes Sánchez Martínez (principal) y Alma Alicia Benítez } \\
\text { Pérez (igual) }\end{array}$ \\
\hline $\begin{array}{l}\text { Escritura - Preparación del } \\
\text { borrador original }\end{array}$ & $\begin{array}{l}\text { Alma Alicia Benítez Pérez (principal), Aída María Castañeda } \\
\text { Rodríguez Cabo (apoyo) y Reyes Sánchez Martínez (apoyo) }\end{array}$ \\
\hline $\begin{array}{l}\text { Escritura - Revisión y } \\
\text { edición }\end{array}$ & $\begin{array}{l}\text { Alma Alicia Benítez Pérez (principal), Aída María Castañeda } \\
\text { Rodríguez Cabo (igual) }\end{array}$ \\
\hline Visualización & $\begin{array}{l}\text { Alma Alicia Benítez Pérez (principal), Aída María Castañeda } \\
\text { Rodríguez Cabo (apoyo) }\end{array}$ \\
\hline Supervisión & Alma Alicia Benítez Pérez \\
\hline $\begin{array}{l}\text { Administración de } \\
\text { Proyectos }\end{array}$ & $\begin{array}{l}\text { Alma Alicia Benítez Pérez (principal) y Reyes Sánchez } \\
\text { Martínez (igual) }\end{array}$ \\
\hline Adquisición de fondos & Alma Alicia Benítez Pérez \\
\hline
\end{tabular}

